\title{
Correspondence
}

\section{Epidural anesthesia and splanchnic perfusion}

To the Editor:

Piper et al. reported that during and after aortic surgery, gastric intramucosal $\mathrm{pH}(\mathrm{pHi})$ showed a similar decrease from baseline in patients with and without epidural anesthesia. $\mathrm{CO}_{2}$-gap was also comparable between groups. ${ }^{1}$

These findings are in disagreement with the majority of studies on the effects of epidural anesthesia on splanchnic perfusion during abdominal surgery. Most studies reported beneficial effects of epidural anesthesia, resulting in increased regional blood flow or gastric $\mathrm{pHi}^{1-3}$

Experimental work using intravital microscopy also demonstrated that thoracic epidural anesthesia increases gut mucosal blood flow. ${ }^{4}$ Others reported that during lumbar epidural blockade, intestinal vasoconstriction resulted due to a compensatory increase in splanchnic sympathetic activity. Therefore, beneficial changes in gastrointestinal mucosal blood flow (and $\mathrm{pHi}$, or $\mathrm{CO}_{2}$ gap) cannot be expected if the epidural results in anesthesia outside the area of interest.

Usually, investigators who demonstrated evidence for improved splanchnic blood flow during epidural anesthesia performed measurements inside the anesthetized region. ${ }^{2-4}$ In the study by Piper et al., epidural catheters were inserted at L3 $-4 .^{1}$ Therefore, the anesthetic block did not include those sympathetic nerves that supply the gastric wall. The site of $\mathrm{pHi}$ measurement and the location of epidural anesthesia were different, thus explaining why epidural anesthesia failed to produce beneficial effects on gastric intramucosal $\mathrm{pHi}$, or $\mathrm{CO}_{2}$ gap.

Obviously, the authors missed this important point. With all their patients having received lumbar epidural anesthesia only, the conclusion that epidural bupivacaine has no effect on splanchnic microvascular hemodynamics and $\mathrm{pHi}$ was inappropriate.

Andreas Sielenkämper MD MSC

Gerhard Brodner MD PhD

Hugo Van Aken MD PhD

References

1 Piper SN, Boldt J, Schmidt CC, Maleck WH, Brosch C, Kumle B. Hemodynamics, intra-mucosal $\mathrm{pH}$ and regu- lators of circulation during perioperative epidural analgesia. Can J Anesth 2000; 47: 631-7.

2 Kapral S, Gollmann G, Bachmann D, et al. The effects of thoracic epidural anesthesia on intraoperative visceral perfusion and metabolism. Anesth Analg 1999; 88: 402-6.

3 Müller M, Schück R, Erkens U, Sticher J, Haase C, Hempelmann $G$. Influence of lumbar peridural anaesthesia on tissue-pO2 in the human colon. (German) Anästhesiol Intensivmed Notfallmed Schmerzther 1995; 30: 108-10.

4 Sielenkämper $A W$, Eicker $K$, Van Aken $H$. Thoracic epidural anesthesia increases mucosal perfusion in ileum of rats. Anesthesiology 2000; 93: 844-51.

5 Hogan QH, Stekiel TA, Stadnicka A, Bosnjak ZJ, Kampine JP. Region of epidural blockade determines sympathetic and mesenteric capacitance effects in rabbits. Anesthesiology 1995; 83: 604-10.

\section{REPLY:}

Sielenkämper et al. criticize two aspects of our study: firstly, that the results with respect to gastric $p H$ were foreseeable and thus our study was superfluous. Secondly, they assume that we concluded that "epidural bupivacaine has no effect on splanchnic microvascular hemodynamics and $p H i$ " and they emphasized that this conclusion is inappropriate.

Concerning their first comment, they base their argument on theoretical assumptions on the effect of lumbar vs thoracic epidural anesthesia (EA) on splanchnic perfusion and on four studies, of which two were done in sham-operated animals and two during intestinal surgery in humans. These studies are definitely different from the aortic surgery setting (where patients present with vascular pathology and undergo aortic cross-clamping) and, consequently, cannot be used to predict the results of our trial. Furthermore our study did not focus on gastric $p H$, but analysed regulators of circulation and hemodynamic responses as well. Publications on EA and regulators of circulation in aortic surgery are rare. ${ }^{1-4}$ At the time the trial was designed, no study on the influence of $E A$ on gastric $p H$ in aortic surgery had been published. A single other article has focussed on these patients since then. ${ }^{5}$ Väisänen et al. reported similar results concerning the inability of thoracic EA to influence gastric $\mathrm{pH}$.

Concerning their second criticism, Sielenkämper et al. misquote us. We wrote: "It is concluded that periop- 